\title{
Design of a Fast and Non-Dissipative Equalization Method for Li-ion Battery Pack
}

\author{
Tao yin-jiao ${ }^{1,}$, Chen hai-jin ${ }^{1, b, *}$ \\ ${ }^{1}$ Nantong University, Nantong 226000, China

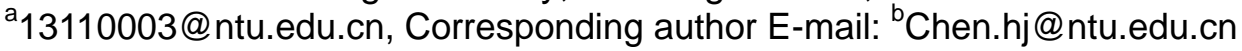

Keywords: Li-ion battery pack; non-dissipative equalization technique; boost circuit; MOSFET switch

\begin{abstract}
The consistency problem of Li-ion battery pack often affects its service life during the usage. To research the equalization techniques of Li-ion batteries can reduce the consistency problem. This paper presented a non-dissipative balancing method using the pulse transformer to drive a couple of MOSFETs that acts as bi-directional switches. Voltages of batteries are sampled using a high common-mode voltage difference amplifier. The result of simulation and experiment showed that this method can reach a fast balancing speed and a high energy transfer efficiency.
\end{abstract}

\section{Introduction}

With the development of the industry, the advantages of Li-ion batteries are showed gradually as the new generation of energy. The traditional Li-ion batteries such as Lithium iron phosphate battery, have the cell voltage between in 2.8-4 V. It cannot meet the requirements of the high voltage and large power in practical industrial application. Therefore, it always uses several batteries connected in series as a power battery pack to use $\mathrm{e}^{[1]}$. Considering the difference of self-discharging rate, the difference of using environment, and the difference of production technology of each battery, the inconsistency of the battery pack will be getting worse, what's more, the available capacity of a battery pack is always determined by the worst battery which has the minimum available capacity of all ${ }^{[2]}$. Therefore, study a reliable balance technology for Li-ion battery pack is an important guarantee for safety ${ }^{[3]}$.

In the existing balance technologies, the balance speed is often slow at later balancing period when using a super capacitor. The balance accuracy cannot be guaranteed when using the transformer due to the consistency problem of the output winding. In the commonly used DC-DC balance methods, the loss of the switching devices is often large ${ }^{[4]}$, the isolated driving circuits is often complex ${ }^{[5]}$, these problems increase the cost of the circuit. This paper studied a non-dissipated equalization technology based on the BOOST-BUCK circuit which transfers energy between any two batteries. This plan can reduce resistance loss by using a pulse transformer to drive a couple of MOSFET switches, shorten the balance time, and is very suitable for high voltage and large power applications.

\section{The principle and analysis of the Equalization Method}

The proposed equalization circuit is shown in Fig. 1, the $\left\{B_{n}\right\}$ are represented as the series Li-ion batteries, the $\left\{\mathrm{S}_{\mathrm{n}}\right\}$ are represented as the switches connected to the batteries. $\mathrm{L}_{\mathrm{b}}$ is the boost inductor of the BOOST circuit, $\mathrm{S}_{\mathrm{b}}, \mathrm{S}_{\mathrm{a}}$ are control switches, $\mathrm{D}$ is a freewheeling diode, $\mathrm{C}$ is an energy storage capacitor. The working process can be divided into three stages: $t_{0} \sim t_{1} 、 t_{1} \sim t_{2}$ 、 $\mathrm{t}_{2} \sim \mathrm{t}_{3}$. 


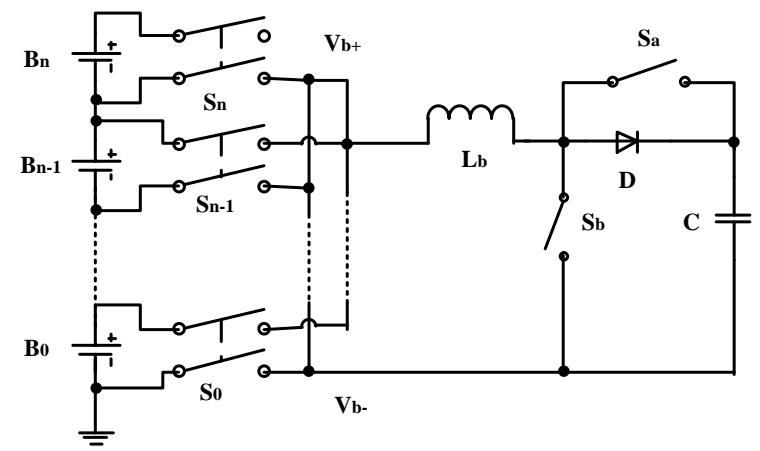

Fig. 1 Equalization circuit

(1) $t_{0} \sim t_{1}$ : The circuit will be connected to the battery whose open circuit voltage is highest among the battery pack, and it will work as a boost circuit. The $\mathrm{S}_{\mathrm{b}}$ is on, $\mathrm{S}_{\mathrm{a}}$ is off, the current $\mathrm{i}_{\mathrm{b}}$ will rise, the voltage of capacitor $C$ will remain. The equivalent circuit is shown in Fig. 2(a), the current in the circuit is shown as follows:

$$
i(t)=\frac{v_{\text {max }}}{R_{e q}}\left(1-e^{-\frac{R_{e q} t}{L_{b}}}\right)
$$

The $R_{\text {eq }}$ is the equivalent resistance of the circuit, and the $V_{\max }$ is the open circuit voltage of the battery which is of highest capacity.

(2) $t_{1} \sim t_{2}$ : The circuit is connected to the highest voltage battery. Assuming the initial voltage of the capacitor $\mathrm{C}$ is $\mathrm{V}_{\mathrm{c} 0}, \mathrm{~S}_{\mathrm{b}}$ should be off until $\mathrm{t}_{1}\left(\mathrm{~S}_{\mathrm{a}}\right.$ is remained on), the current will be decreased gradually before $t_{2}$, and become zero until $t_{2}$. At this time the voltage between the capacitor will rise to maximum value $V_{\text {peak }}$, the equivalent circuit is shown as Fig. 2(b).

(3) $t_{2} \sim t_{3}$ : The circuit will charge the battery whose open circuit voltage is the lowest of the pack, and the $\left\{S_{n}\right\}$ will be changed to connect with it. At the time of $t_{2}$, the $S_{a}$ is on, the Capacitor will release energy to the battery which need balanced, the voltage of capacitor $C$ will drop from $V_{\text {peak }}$ to $V^{\prime}{ }_{c 0}$. Because the transferred energy is very small in one balance cycle, so the $\mathrm{V}^{\prime}{ }_{\mathrm{c} 0}$ can be equaled with $\mathrm{V}_{\mathrm{c} 0}$ between this time. The equivalent circuit is shown in Fig. 2(c). In this period, the circuit is equivalent to the RLC resonant circuit ${ }^{[4]}$, and the resonant period can be calculated as follows:

$$
\mathrm{T}=2 \pi \sqrt{\mathrm{L}_{\mathrm{b}} \mathrm{C}}
$$

Therefore, the values of Inductor $\mathrm{L}_{\mathrm{b}}$ and capacitor $\mathrm{C}$ determine the switching frequency and duty cycle of $S_{a}$.

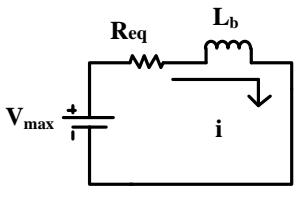

(a)

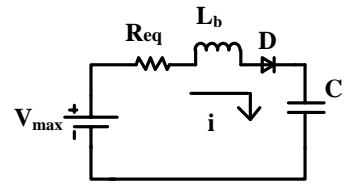

(b)

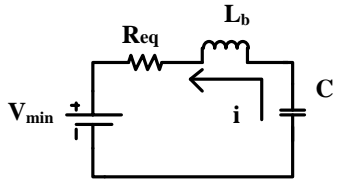

(c)

Fig. 2 The equivalent circuit of the three periods

\section{Design of balance system}

Fig. 3 shows a block diagram of the whole battery management system. It uses a STM32F100C8 as the main controller. In balancing process, the main controller will send signals to control the switches, sample the voltages of each cell in the battery pack by turns, and make comparisons of these sample voltages. And then, changing switches between the highest one and lowest one to transfer energy. In addition, a fuse is connected in series in the circuit in case of the miss operation of the boost switch $S_{b}$, which may cause safety accidents. 


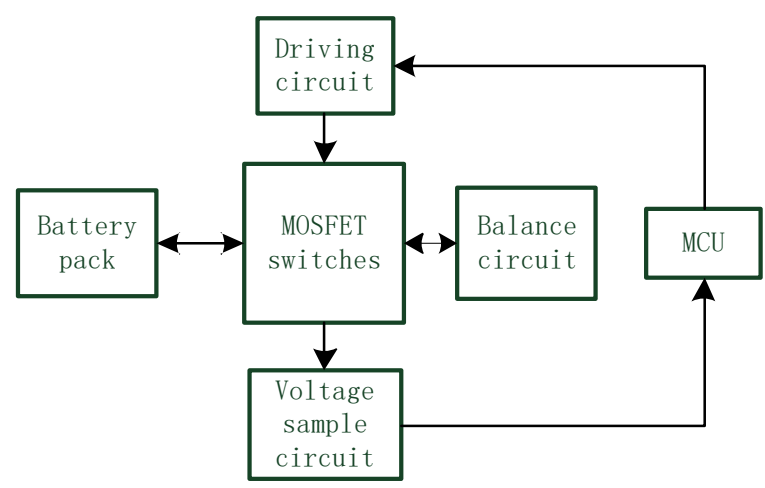

Fig. 3 Block diagram of the balance system

MOSFET switch and drive circuit. Because of its body diode, the MOSFET is usually used as a single directional switch device. In this paper, we will connect the source electrodes in series to realize a bi-directional MOSFET switch. The N-channel MOSFET DTS6400 from Din-Tek are used as all switches, it has the advantage of high continuous drain current and small conductive resistance. The voltages of source and gate electrode of the NMOS are floating, so an isolated MOSFET driving circuit must be added. In this paper, a pulse transformer is used to drive a couple of MOSFET switches. The driving circuit is shown in Fig. 4. In addition, when the MOSFET is turning off, the pulse transformers can provide a negative voltage to accelerate the gate-source capacitor to discharge ${ }^{[7]}$.

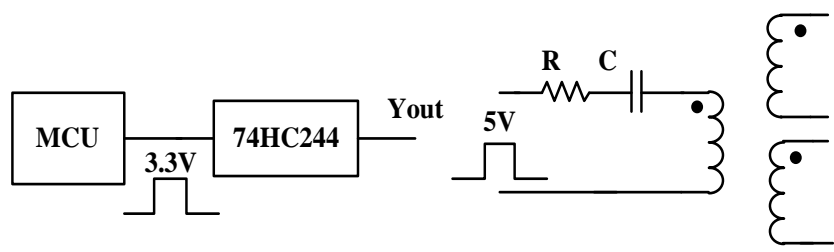

Fig. 4 MOSFET isolated driving circuit

Due to the limited driving ability of the GPIO port, the 74HC244 chip is added between the MCU and the pulse transformer.to increase the driving voltage and current. In the driving circuit, a capacitor and a resistant is added in series in the primary side of the transformer, which can isolate the DC voltage and prevent resonance problem.

Cell voltage sample circuit. The critical point of sampling voltage for each cell in Lithium-ion battery pack is to eliminate the high common voltage and increase the accuracy of the sample voltage for each battery. The voltage sample circuit in this paper is shown in Fig. 5. A high common mode amplifier called INA148UA from TI is used to eliminate the high common voltage. And then, at the common-mode output side and the $2.5 \mathrm{~V}$ reference voltage output side, a subtraction circuit is used to get the result of $\mathrm{V}_{\mathrm{o3}}$, the value can be calculated as follows:

$$
V_{o 3}=\frac{2 R}{R} \cdot\left(V_{o 1}-V_{o 2}\right)=2\left(V_{o 1}-V_{o 2}\right)
$$

The precision of all the resistors in the sample circuit is $0.1 \%$. A filter capacitor $C$ is added in parallel to eliminate the interference due to high common-mode voltage.

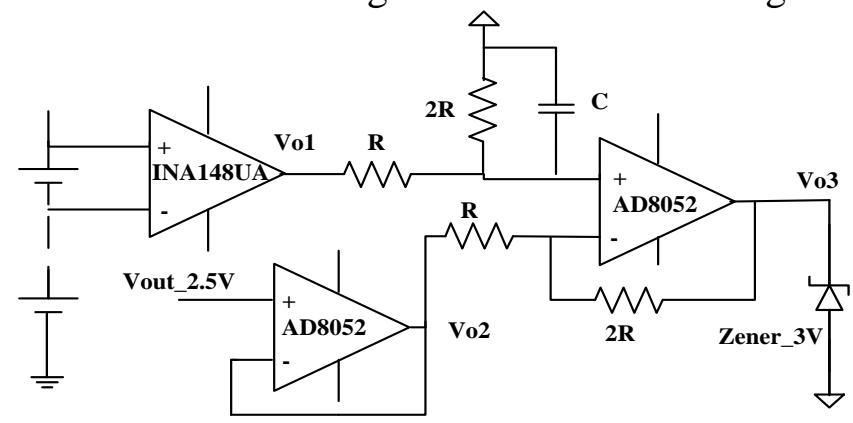

Fig. 5 Voltage sample circuit

Balance control strategy. Considering the inductance value and limited current of a conductor, a CDRH125 inductor is selected as $\mathrm{L}_{\mathrm{b}}$, the value is of $10 \mu \mathrm{H}$ and the limited current is $4 \mathrm{~A}$. The 
capacitor $\mathrm{C}$ in Fig. 1 is replaced by two $4.7 \mu \mathrm{F}$ paralleled capacitors. According to formula(1) and (2) the conduction time of $S_{a}$ and $S_{b}$ can be calculated to be $31 \mu s$ and $15 \mu \mathrm{s}$, and the continued flow time of diode $\mathrm{D}$ can be tested as $12 \mu \mathrm{s}$.

In the scheme, the voltage sample circuit and the balance circuit use common switches $\left\{\mathrm{S}_{\mathrm{n}}\right\}$, so that a pre-charge process of the capacitor $\mathrm{C}$ in Fig. 1 before balancing is needed. The flow chart of this system is shown in Fig. 6. The voltage of each cell is sampled every 1 second, and the balance circuit is stopped while sampling voltage. Assuming a threshold value called $V_{t}$ is setted, if the maximum difference voltage in each cells is larger than $\mathrm{V}_{\mathrm{t}}$ after sampling, then the balance circuit will start to work. Otherwise, the balance system should wait for the time interrupted.

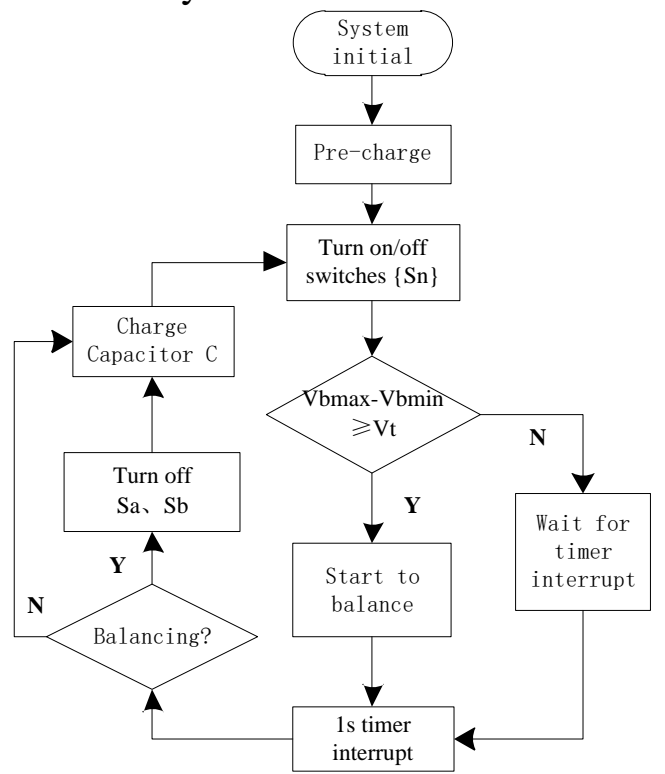

Fig. 6 Flow chart of the system

\section{Experiment result and analysis}

PSPICE simulation results. In order to verify the feasibility of this scheme, the circuit is verified in PSPICE environment according to Fig. 1. Assuming the equivalent resistance is $0.7 \boldsymbol{\Omega}$, we can get the value $\mathrm{V}$ which means the voltage of the battery, the current $\mathrm{i}$ which means the current of the inductor $\mathrm{L}_{b}$ and the voltage $\mathrm{V}_{\mathrm{c}}$ which means the voltage of the capacitor by simulation in PSPICE software. The simulation results is shown in Fig. 7 . In the first period $t_{0} \sim t_{1}$, the current in inductor is rising to the maximum value, in the second period $t_{1} \sim t_{2}$, the current drop to zero, in the third period, the circuit is charging to the lowest voltage battery.

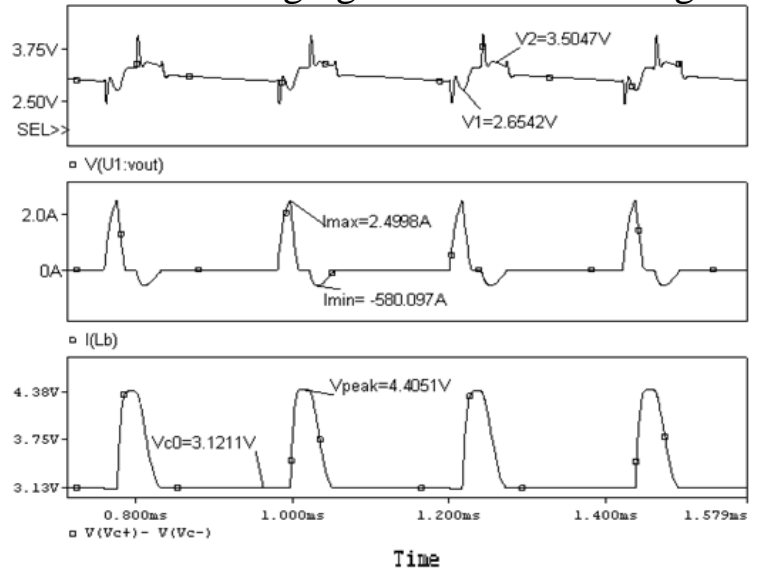

Fig. 7 The simulation result

Hardware circuit and experimental results. The hardware circuit, shown in Fig. 8(a) is implemented and tested. The waveform of the inductor current and the capacitor voltage is shown in Fig. 8 (b), and the current changes from $-0.6 \mathrm{~A}$ to $2.4 \mathrm{~A}$, the voltage changes from $3 \mathrm{~V}$ to $4.5 \mathrm{~V}$. Fig. 
8(c) shows the comparison between the sample voltage and the actual voltage which measured by a Keithley digital meter.

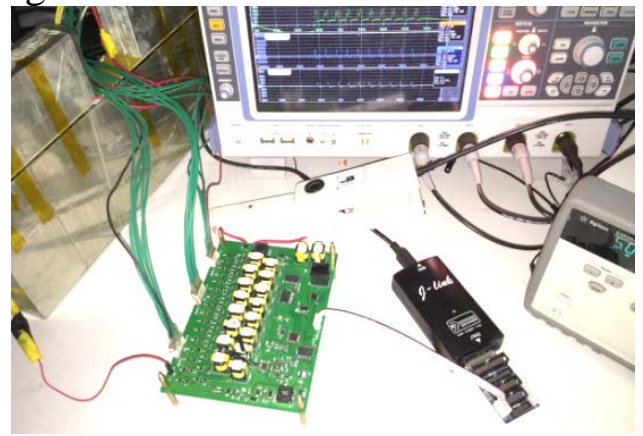

(a)

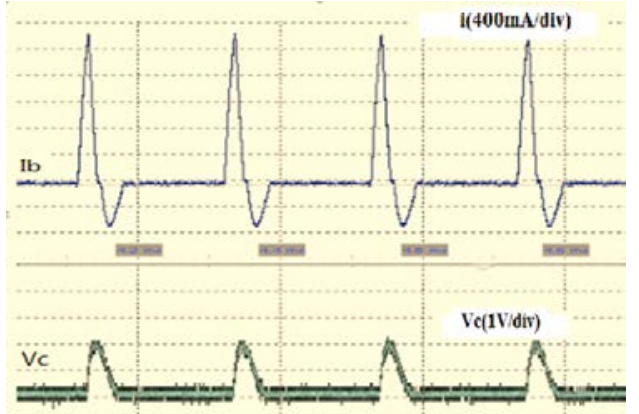

(b)

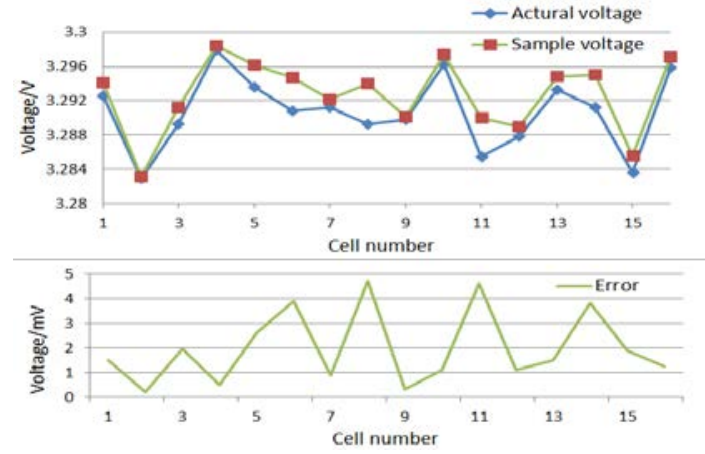

(c)

Fig. 8 Hardware circuit and test result

Analysis of experimental result. After performing an experiment on $16 \mathrm{Li}$-ion batteries with $50 \mathrm{Ah}$, the error is within $\pm 5 \mathrm{mV}$ between the sample voltage and the actual voltage. Finally, by recording the voltages of the 16 batteries in balancing process, the maximum difference in the battery pack can be calculated in Fig.9. The result shows when the maximum difference between the battery pack can be reduced from $15 \mathrm{mV}$ to $5 \mathrm{mV}$ in 15 minutes.

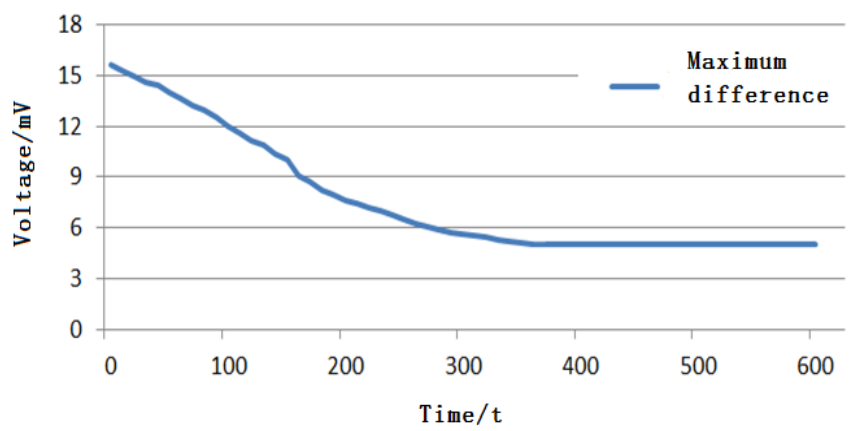

Fig. 9 The changes of the maximum difference in cells

\section{Conclusion}

In this paper, we designed a Li-ion battery equalization scheme, which based on the BOOST-BUCK circuit. By using a high common mode amplifier, we implemented a high precision voltage acquisition method. By using MOSFETs as bi-directional switches and pulse transformers as isolated drivers, Finally, experiment results shows the feasibility of this scheme, and proved that this method can transfer energy between batteries quickly and effectively.

\section{References}

[1] Kim. M Y, Kim. C H, Kim. J H, A Chain Structure of Switched Capacitor for Improved Cell Balancing Speed of Lithium-Ion Batteries, J. IEEE Transactions on Industrial Electronics, 2014, 61(8) 3989-3999. 
[2] Phung. T H, Collet. A, Crebier. J C. An Optimized Topology for Next-to-Next Balancing of Series-Connected Lithium-ion Cells, J. Power Electronics IEEE Transactions on, 2011, 29(9) 1374-1381.

[3] Li. S, Mi. C C, Zhang. M. A High Efficiency Active Battery Balancing Circuit Using Multi-Winding Transformer, J. IEEE Transactions on Industry Applications, 2011, 49(1) 198 - 207.

[4] Michal V. Peak-Efficiency Detection and Peak-Efficiency Tracking Algorithm for Switched-Mode DC-DC Power Converters, J. IEEE Transactions on Power Electronics, 2014, 29(12) 6555-6568.

[5] Shang. Yunlong, Zhang. Chenghui, Cui. Naxin, Josep M.Guerrero. Acell-to-Cell Battery Equalizer With Zero-Current Switching and Zero-Voltage Gap Based on Quasi-Resonant LC Converter and Boost Converter, J. IEEE Trans. PowerElectron, 2015, 30(7), 3731- 3747.

[6] Miu. Hongfei, Liang. Qifeng, Peng. Jianyu. The Parameters Design for Power MOSFET Driver Circuit Based on Transformer Isolated, J. Telecom Power Technologies, 2012, 29(3) 31-32. 